\title{
Thyroid Hormone Resistance: Multicentrical Case Series Study
}

Authors Maria Angeles Santos Mataํ, Ana Belen Ariza Jimenez ${ }^{2}{ }^{\mathbb{D}}$, Francisco Macias Lopez ${ }^{1}$, Carmen de la Camara Moraño

\author{
Affiliations \\ 1 Hospital Maternoinfantil de Jerez, Cadiz, Spain \\ 2 Hospital Universitario Reina Sofía, Córdoba, Spain
}

Key words

resistance to thyroid hormone, thyroid receptor, symptoms, diagnosis, treatment

received 25.08.2021

accepted after revision $\quad$ 14.12.2021

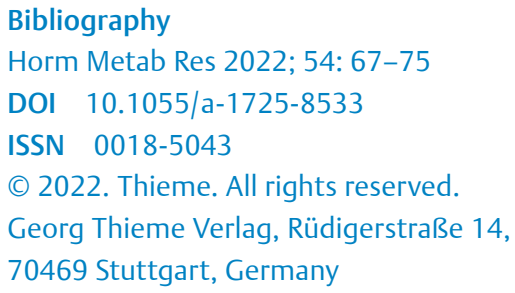

\section{ABSTRACT}

Resistance to thyroid hormone syndrome (RTHS) is defined as increased thyroxine and triiodothyronine associated with normal or increased thyrotropin. This is usually due to a pathogenic variant of the gene coding for thyroid hormone receptor $B$ (THRB). THRB is a rare genetic disorder characterized by an altered response of target tissue to the thyroid hormone action. Retrospective cross-sectional observational study with diagnosis of RTHS evaluated in secondary and tertiary hospitals for 6 years, from 2014 to 2020, in order to describe variables including age, sex, anthropometric data, clinical and biochemical characteristics of patients, who were divided according to age, in a pediatric group from 0 to 14 years (index cases), and an adult group composed of adult relatives of index cases. $A$ molecular analysis of the THRB gene was performed. The total retrospective cohort included 7 pediatric patients and 15 adults. We found 22 cases with a clear male predominance (14/22). Mean age is 24.8 years old ( 22 days- 70 years). Patients were referred because of symptoms $18.2 \%$ (4/22), analysis results $22.7 \%$ (5/22), or familial study $59.1 \%$ (13/22). About $31.8 \%$ (7/22) cases show goiter, $31.8 \%$ (7/22) sympathetic symptoms and $13.6 \%$ (3/22) abnormalities in behavior. In most cases, $77.3 \%,(17 / 22)$ show familial background of thyroid abnormalities. It is important to remark that $18.2 \%$ (4/22) relatives received previous incorrect treatments such as thyroidectomy, because of wrong diagnosis. In conclusion, a better understanding of RTHS, its prompt molecular diagnosis and genetic counseling, could avoid unnecessary tests and inappropriate treatments.

\section{Introduction}

Resistance to thyroid hormone (RTH) [1] is a genetic disorder characterized by an impaired responsiveness of target tissues to the action of the thyroid hormone. There is an abnormal increase of serum thyroxine (T4) levels generally accompanied by elevated serum triiodothyronine (T3), with non-suppressed (normal, or even elevated) serum thyroid-stimulating hormone (TSH) levels.

Resistance to thyroid hormone is rare, with an estimated incidence of 1:40 000 births [2], although accurate data is difficult to obtain. The classic form is, in most cases, due to mutations in the Thyroid Hormone Receptor $\beta$ (THRB) gene, although there are also alterations in the cellular transport of $\mathrm{T} 4$ and $\mathrm{T} 3$, and in the conversion from T4 to T3 mediated by deiodinases $[3,4]$.

The effects of the thyroid hormone are mediated by a receptor encoded by separate genes: THRA (thyroid hormone receptor $\alpha$ ) gene, coding for thyroid hormone receptor alpha (TR $\alpha)$, isoforms 1 and 2, located in 17q11.2; and the THRB gene, isoforms 1 and 2, located in 3p24.2 [5].

Currently, according to the THRB gene, 120 mutation sites have been reported, most of which are located in the "hotspot" region. These are carboxyl terminal ligand binding regions of TRB encod- 
ed by exon 7 to 10 [6]. Mutated proteins provoke a reduced affinity for T3 and/or an impaired interaction with the cofactors involved in their transcriptional machinery. Thus, dominant negative inhibition of wild-type receptors by the mutant THRB form is the basis of the disorder [7]. Although some cases have been described as being caused by the mutation receptor $\alpha 1$ and $\alpha 2[1,8]$, the majority of them (80-85\%) are due to heterozygous mutation, although some homozygosis mutations have also been described [9].

RTH- $\beta$ is an autosomal dominant disease, which has affected individuals who are heterozygous for mutant allele, although recessive heritance and $20-25 \%$ of novo mutations have also been described [10]. On the other hand, gene defects remain unknown in $15 \%$ of subjects with a phenotype similar to RTH- $\beta$, called "nonthyroid receptor-resistance to thyroid hormone (TR-RTH)". It is due to mutations in genes, which encode cofactors that interact with receptors $[1,10,11]$.

The first description of RTH was made by Refetoff et al. [12] in a family with congenital deafness, stippled epiphyses, goiter, and abnormal high serum protein-bound iodine. The majority of patients maintain an almost normal serum level, although it is variable among people affected. The most common abnormality is the presence of diffuse goiter (66-95\%), followed by tachycardia 35\% [7]. However, patients with RTH- $\beta$ show very heterogeneous clinical manifestations, being asymptomatic or presenting symptoms from hypothyroidism to hyperthyroidism. Even with the same mutation, due to the distribution of receptor expression, compensatory mechanisms and the effect of previous and/or current treatment, different degrees of peripheral resistance are observed in patients, as well as variable resistance in different tissues of the same individual [9].

Some common symptoms are described in children: short stature, delayed bone age, deafness, language delay, school delay and even intellectual delay. An amount of $70 \%$ show Attention-Deficit Hyperactivity Disorder (ADHD) $[4,13]$.

Thyroid antibodies may be present in $20 \%$ of these patients $[14,15]$. Clinical presentation needs to be differentiated with TSH secreting adenoma, familial hypertyrosinemia, hypalbuminemia, abnormalities in proteins such as albumin and thyroglobulin [10].

The present review was prepared for the purpose of expanding knowledge of RTH- $\beta$ in order to reduce the rate of misdiagnosis.

\section{Ethics Approval}

All procedures followed in both centers were in accordance with the ethical standards of Virgen del Rocio Hospital's Ethics committee (code 12011966). Informed consents before the inclusion of patients were not necessary because of the retrospective design.

\section{Patients and Methods}

Retrospective cross-sectional observational study with diagnosis of RTH evaluated in secondary and tertiary hospitals for 6 years (Jerez Hospital and Reina Sofia University Hospital) from 2014 to 2020 , in order to describe clinical and biochemical characteristics of patients, who were divided according to age, in a pediatric group from 0 to 14 years (index cases), and an adult group composed of adult relatives of index cases. Exclusion criteria were: Alteration of transporter proteins, dysalbuminemia, adenoma, and other genetic etiologies different to RTH- $\beta$, RTH- $\alpha$, or TR-RTH.

In all patients, the diagnosis was based on two biochemical analyses after 12-hour fasting \{T4, T3, TSH; autoimmunity, sex hormone-biding globulin (SHBG), thyroglobulin], thyroid ultrasound, echocardiogram, sellar magnetic resonance imaging (MRI), TRH test, and genetics.

Genetic studies were done through peripheral blood samples collected in ethylenediaminetetraacetic acid (EDTA) tubes. DNA was extracted using Qiagen technology. Structural analysis of THRB gene exons 3-10 and intronic regions were studied by polymerase chain reaction (PCR) amplification. Sequences were analyzed by Termofisher Scientific's SeqScape V3 Software.

During the study, the following variables were collected: sex, age at diagnosis, anthropometric data at birth and at diagnosis, clinical symptoms, biochemical and imaging results, and genetics. Descriptive analysis was done using statistical package $R$, version 4.0.3, through percentages, ranges, and means.

\section{Results}

In the pediatrics group, we found 7 cases with a clear male predominance $(5 / 7)$, which represents $71.4 \%$ of the sample. Mean age is 5.58 years old ( 22 days -14 years). In the adults group, we found 15 patients with a mean age of 44 years old (19-70 years), and male predominance $(9 / 15)$.

Patients were referred for different reasons: $18.2 \%$ (4/22) were referred because of clinical suspicion of RTH (goiter, tachycardia), $22.7 \%$ (5/22) were referred after abnormal T4 and/or TSH levels in biochemical analysis performed due to clinical symptoms not related to RTH (analytical finding), and 59.1\% (13/22) were studied because they were relatives of a patient with a known mutation in the THRB gene (familial study) ( $\triangleright$ Fig. 1). They showed low TSH/ alpha subunit ratio and negative TRH test.

An amount of $31.8 \%$ (7/22) of cases show goiter, $31.8 \%$ (7/22) sympathetic symptoms and 13.6\% (3/22) abnormalities in behavior. Most cases, $77.3 \%$, (17/22) show familial background of thyroid abnormalities, and some of them, which represents $31.8 \%$ (7/22) of the sample, were diagnosed with thyroid hormone resistance through the study of their children. It is important to remark

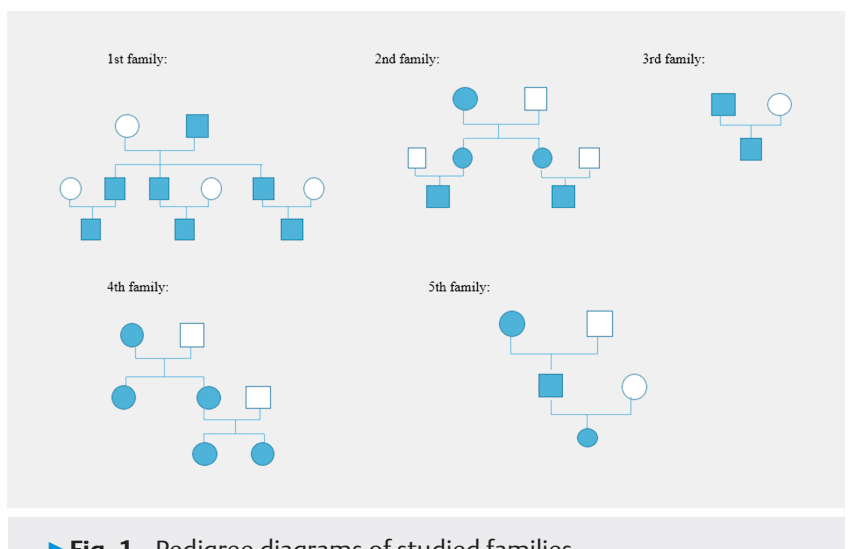

Fig. 1 Pedigree diagrams of studied families. 
that $18.2(4 / 22)$ of relatives received previous incorrect treatments such as thyroidectomy, because of a wrong diagnosis.

Around $4.5 \%$ (1/22) of cases show a low weight at birth. At the time of writing, genetic results for one last family were pending, but they were treated with antithyroid with bad response. They have shown lab tests compatible with thyroid hormone resistance, and the index case is having a good evolution with tetraiodothyroacetic acid treatment. So, nowadays they are compatible with TRRTH ( $\triangleright$ Table 1 and 2).

\section{Discussion}

We found 7 pediatric cases and 15 adults diagnosed with resistance to thyroid hormone, a bigger sample than most publications, especially regarding children [16-18]. In our sample, most reasons for study were in the context of a family study or after obtaining an altered T4 and/or TSH level in a biochemical analysis, as well as in published studies. Although, they referred to biochemical analysis performed due to clinical symptoms unrelated to RTH- $\beta$ [17], while most cases in our sample were performed for clinical symptoms compatible with thyroid abnormalities.

As we can see in our series, as well as in the literature, depending on the tissue, features of thyroid hormone excess and deficiency may coexist, although most individuals have a euthyroid, normal metabolic state at the expense of high thyroid levels [7]. In fact, the syndrome is often misdiagnosed as hyperthyroidism and unnecessarily treated with antithyroid drugs, as occurred with one of our cases, and some patients receive I-thyroxine treatment for apparent hypothyroidism [19]. So, due to their nonspecific symptomatic presentation, these patients can be misdiagnosed if the physician is not familiar with the condition. This can result in frustration for the patient and sometimes unnecessary invasive treatment, such as radioactive iodine ablation or thyroidectomy [20,21], as in our familial cases. Routine neonatal screening based on the TSH assay has a limited role in detecting resistance to thyroid hormone, although it could facilitate the early diagnosis of RTH- $\beta$ in newborns in some cases [16,17]. Therefore, genetic testing of the candidate genes THRB should be performed for diagnosis of resistance to the thyroid hormone in patients with the suggestive clinical phenotype [16] or familial antecedents, because prompt molecular diagnosis and genetic counseling could prevent unnecessary tests and inappropriate treatments such as iodine or surgery [22].

It is important to highlight that we found some pathogenic variants in our sample, which have not been described before, such as c.1348 C > T; p. (Leu450Phe). These pathogenic variants were defined by experimented geneticians according to known databases and experience.

Fetuses born to RTH- $\beta$ mothers without diagnosis and treatment have poor intrauterine and postnatal growth due to gestational hypertension and exposure to an excess of the thyroid hormone, with low birth weight and suppressed postnatal thyroid-stimulating hormone (TSH) [21, 23]. Probably, this is the situation with our case 3 , who showed low neonatal anthropometry. In fact, adult humans and mice without resistance to the thyroid hormone- $\beta$ exposed in utero to high maternal thyroid hormone levels have persistent central resistance to the thyroid hormone. This is likely mediated by the increased expression of D3 in the anterior pituitary, enhancing local T3 degradation [24]. So, it is important that maternal fT4 levels are not above $50 \%$ of the upper limit of normal in RTH- $\beta$ mothers carrying fetuses. This seems to be a prudent approach that prevents the otherwise expected low birth weight and postnatal TSH suppression [23].

On the other hand, we found one case with thyroid hormone resistance and attention deficit hyperactivity disorder. The cognitive phenotype of resistance to thyroid hormone has been reported to be similar to attention deficit hyperactivity disorder. In fact, there were no significant differences with regard to behavior or electrophysiological phenotype, so it is impossible to determine the real cause of that behavior [13].

Regarding the diagnosis, it would be recommendable to conduct a second thyroid function test (TSH, free T4, and free T3) with a different assay, and then screening for a genetic variant by sequencing the genes involved in thyroid hormone regulation, action and transport (THRB, THRA, SECISBP2, SLC16A, ALB, TTR, SERPINA7) $[3,10]$.

Dieu et al. obtained mutation in THRB in $26 \%$ of cases (15/58), while it is the cause that was evident in all our patients and the most frequent cause in the literature $[25,26]$. Furthermore, they found biological interference due to a thyroid hormone serum transport protein variant in $24 \%(14 / 58)$. On the other hand, biological interference was suspected in $26 \%$ of cases without genetic variant, in which the biological discrepancy was not confirmed by a second analytical technique (15/58). Finally, no etiology for the biological discrepancy could be found in $24 \%$ of cases (14/58) [10], as it occurred in one of our cases. They stated that patients in whom biological discrepancy was due to analytic interference were more often asymptomatic, and patients with no identified etiology tended to be older [10], whilst we show one young patient with this condition.

Xiao et al. also included somatostatin suppression test, electrocardiography (ECG), thyroid ultrasonography, magnet resonance imaging (MRI) of the sellar region, vision and hearing at diagnosis [18]. The reason for this is differential diagnoses with TSH-producing pituitary adenoma (TPA) and Familial Dysalbuminemic Hypertyrosinemia (FDH) [26], and that is why we decided to perform an $\mathrm{MRI}$ in some of our cases.

RTH should be suspected in both adults and children with elevated thyroid hormone and not suppressed TSH [27], so we could diagnose adults due to diagnostic in children, as it occurred in our family cases. It is interesting that higher serum TSH levels in RTH $\beta$ patients have been described when compared to those without mutations in beta isoform of the thyroid hormone receptor, but this difference did not extend to free T4 level [26].

According to treatment, compounds with thyromimetic potency but with different biochemical properties compared to T3 may hold therapeutic potential in these syndromes by bypassing defective transporters or binding to mutant T3-receptors. Such thyroid hormone analogues have the potential to rescue thyroid hormone signaling. So, the application of 3,3',5-triiodothyroacetic acid (Triac) in resistance to thyroid hormone due to defective TR $\beta$ and the role of 3,5-diiodothyropropionic acid (DITPA), 3,3',5,5'-tetraiodothyroacetic acid (Tetrac) and Triac in MCT8 deficiency are really useful $[3,28]$, as we have shown in our cases. 
节

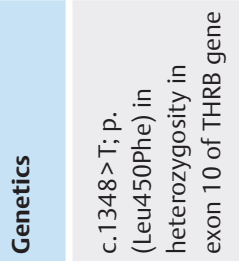

운

之

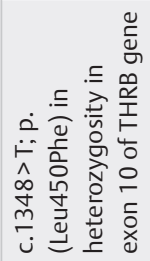

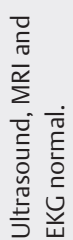

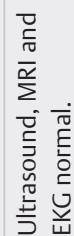

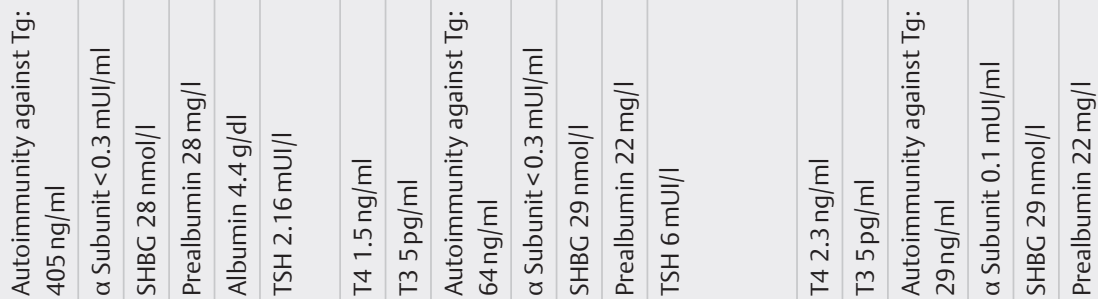

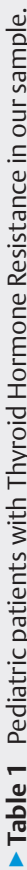

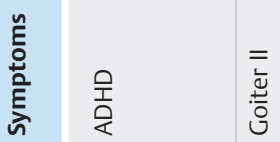

우

우

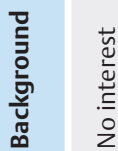

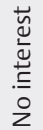

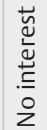

๗ั $\Sigma$

$\Sigma$$$
\frac{\sqrt{2}}{\frac{0}{\pi}} \frac{\sqrt{0}}{\square}
$$

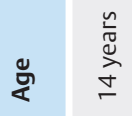

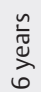

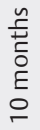




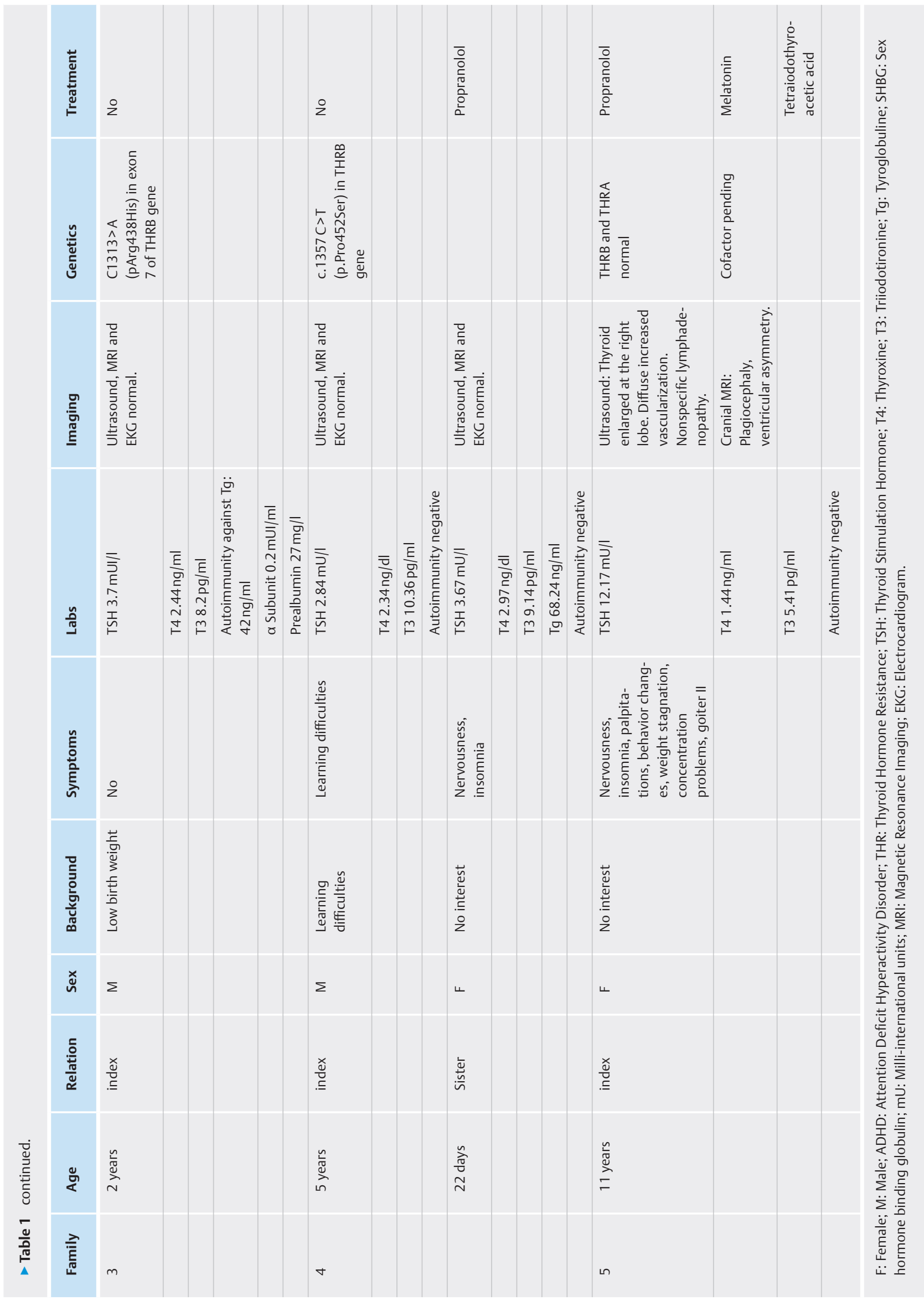




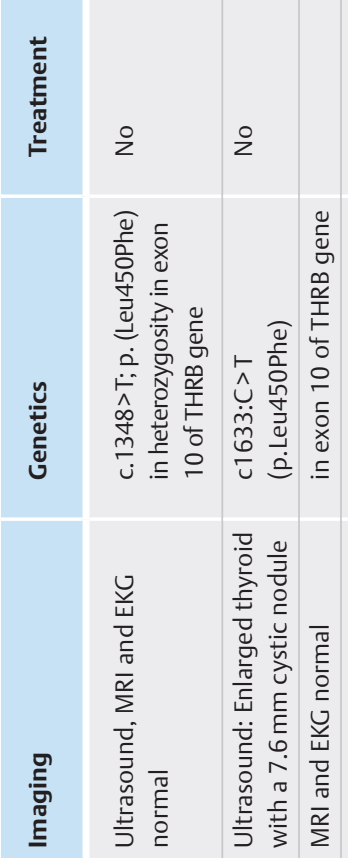

$\stackrel{\circ}{2}$
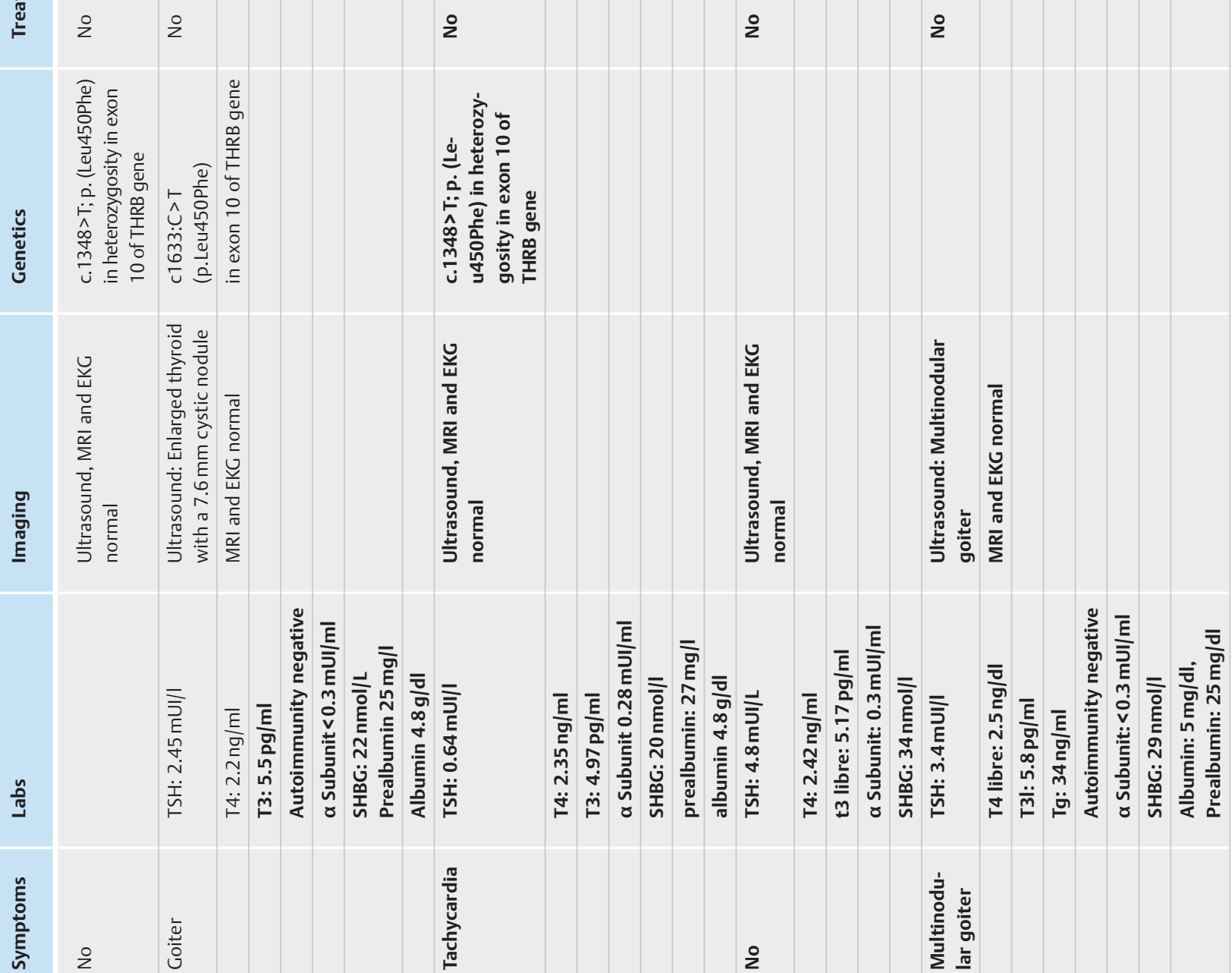

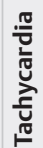

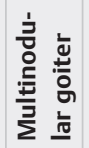

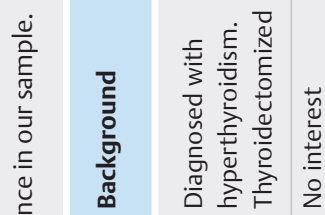
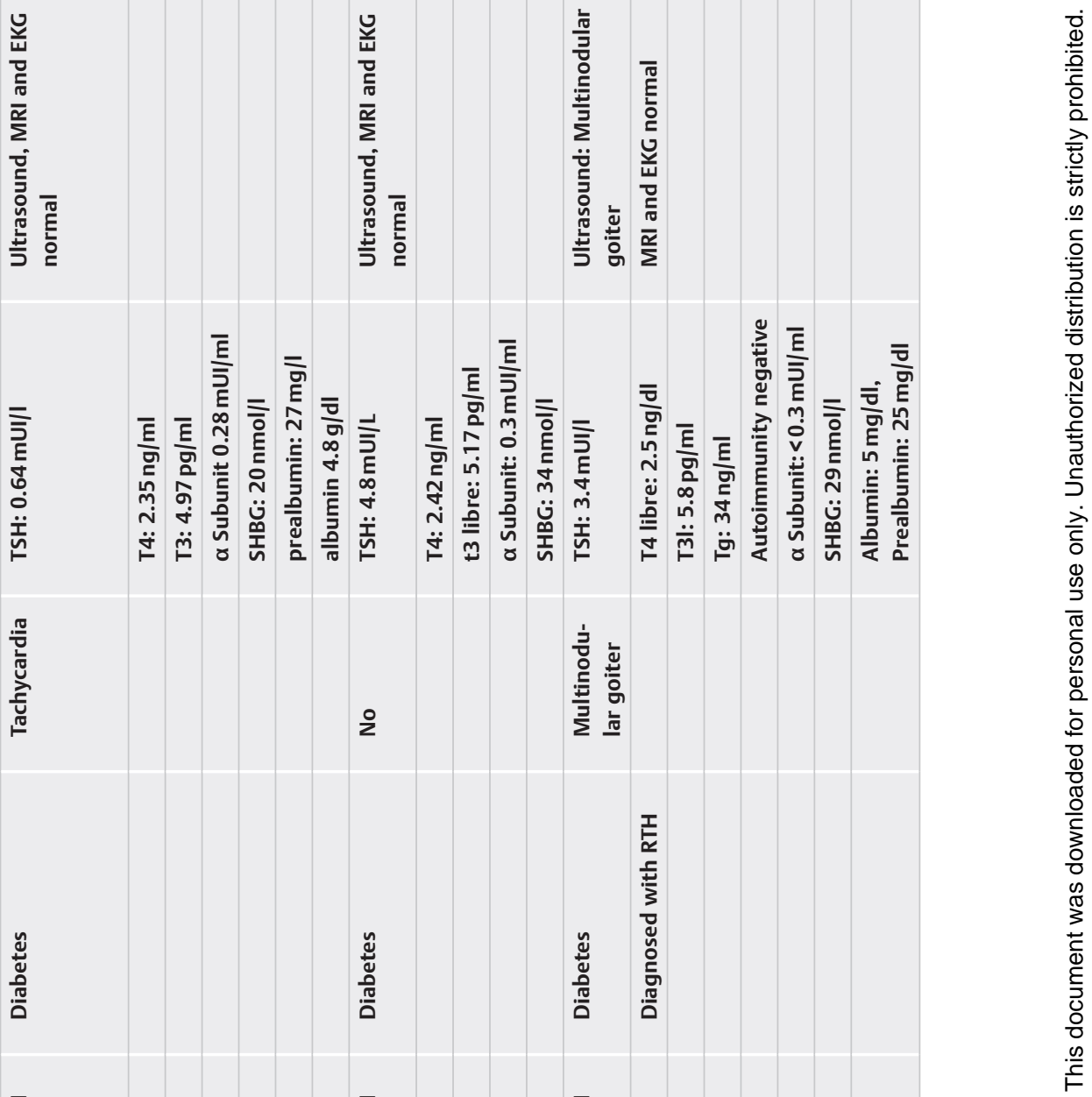

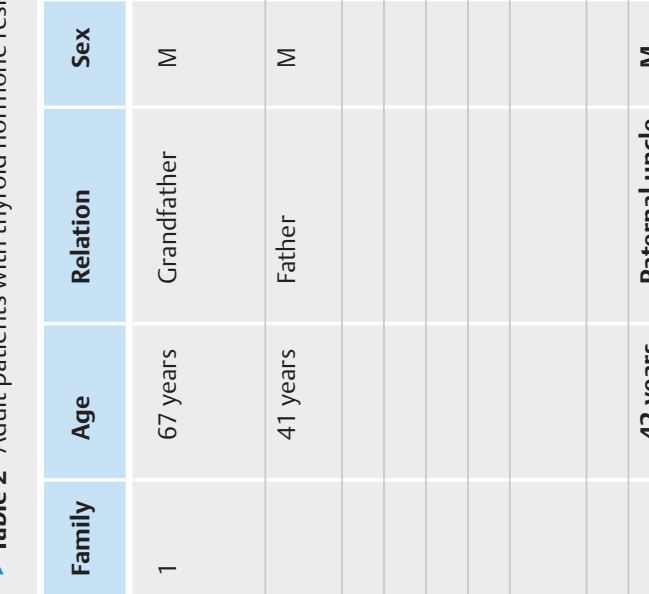



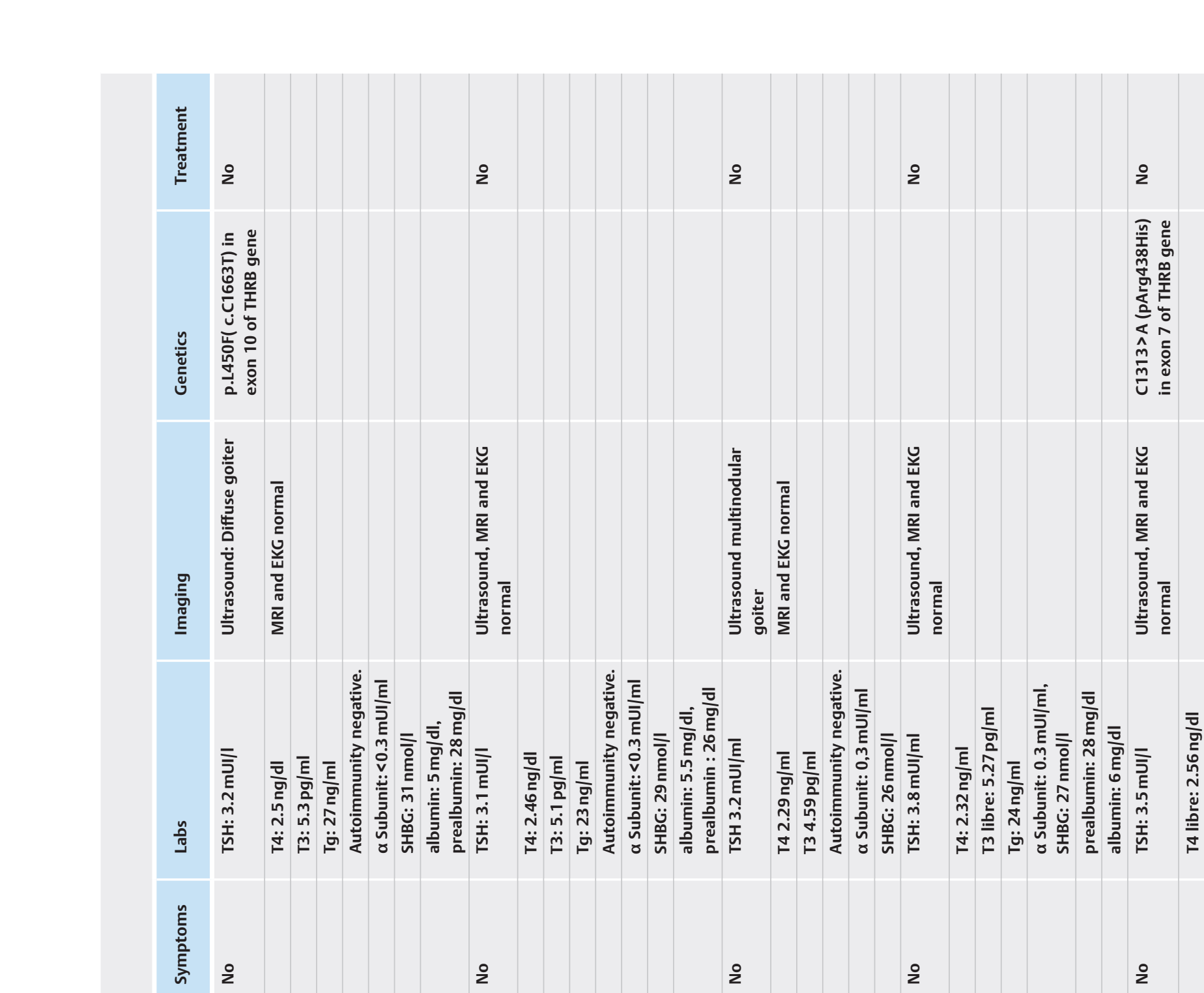

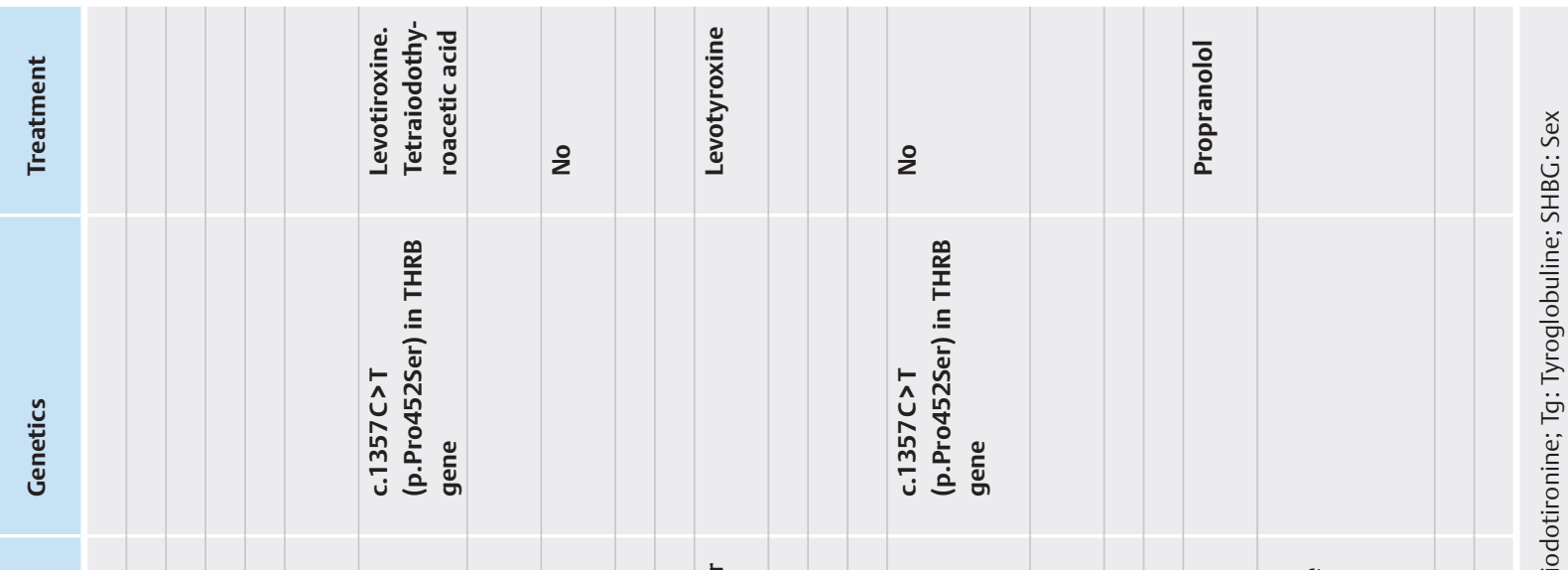

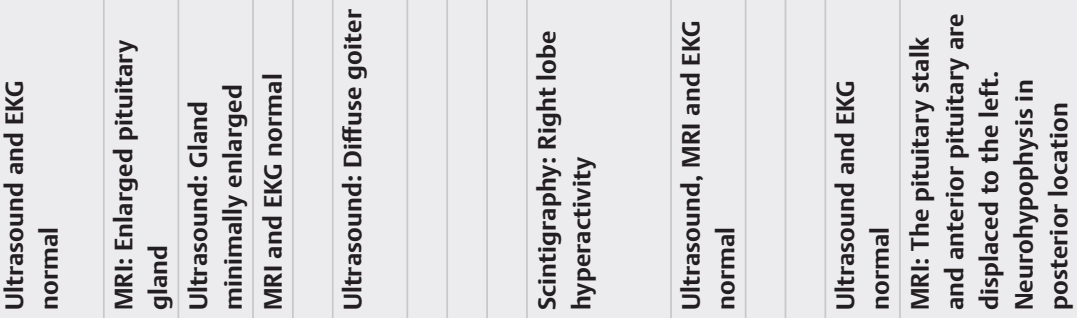

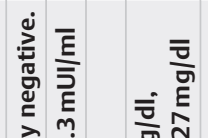

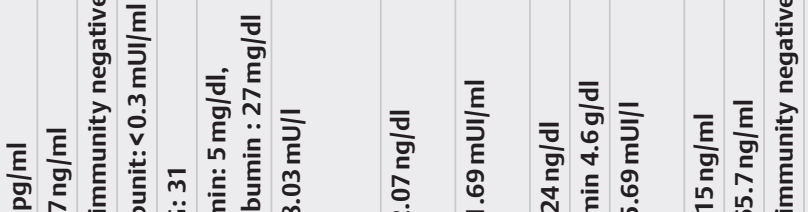

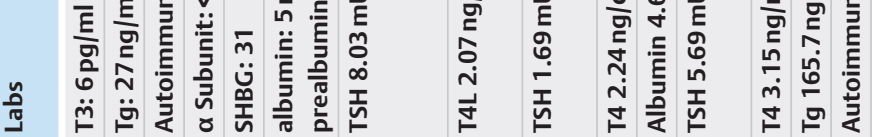

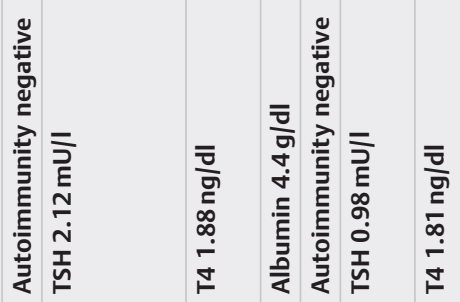

气ิ

竞

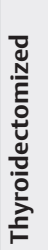

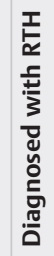

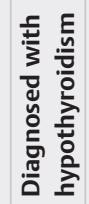

离

๖ัญ

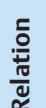

学

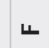

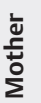

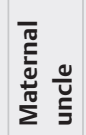

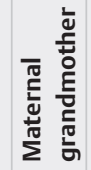

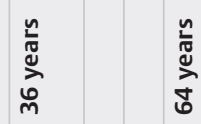

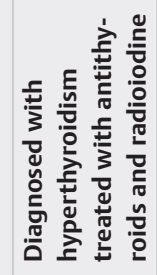

ᄂ

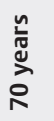

in
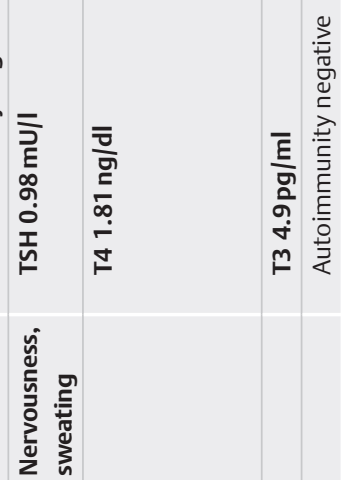

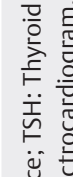

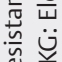

듬

응 끌
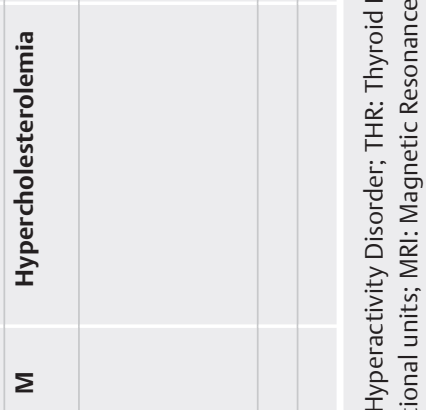

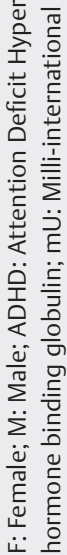


In conclusion, it is necessary to think about resistance to thyroid syndrome in cases of patients with elevated free T4 and T3 concentrations, with normal or inadequately elevated TSH, in the absence of acute illness or drugs, to diagnose it. Prompt molecular diagnosis and genetic counseling could prevent unnecessary tests and inappropriate treatments.

\section{Author Contributions}

M. Angeles Santos Mata has concepted the idea, reviewed literature, and written part of the article. Ana B. Ariza Jimenez has analyzed the data, reviewed literature, written part of the article, and checked final article. Francisco Macias Lopez and Carmen de la Camara Moraño have collected patients.

\section{Acknowledgements}

We acknowledge Ben Nickless and Barbara Samo for their help in translation into English.

\section{Conflict of Interest}

The authors declare that they have no conflict of interest.

\section{References}

[1] Refetoff S, Bassett JHD, Beck-peccoz P et al. Classification and proposed nomenclature for transport, and metabolism. ] Clin Endocrinol Metab 2014; 99: 768-770

[2] LaFranchi SH, Snyder DB, Sesser DE et al. Follow-up of newborns with elevated screening T4 concentrations. J Pediatr 2003; 143: 296-301

[3] Williams GR, Boelen A, Refetoff S. 13th International workshop on resistance to thyroid hormone and thyroid hormone action. Thyroid 2018; 28: 690-691

[4] Bernal J. Sindromes de resistencia a las hormonas tiroideas. Endocrinol y Nutr 2011; 58: 185-196.Available from: http://www.elsevier.es/es/ linksolver/ft/pii/S1575092211000702

[5] Lazar MA. Thyroid hormone receptors: multiple forms, multiple possibilities. Endocr Rev 1993; 14: 184-193

[6] Agrawal NK, Goyal R, Rastogi A et al. Thyroid hormone resistance. Postgrad Med J 2008; 84: 473-477

[7] Pappa T, Refetoff S. Human genetics of thyroid hormone receptor beta:resistance to thyroid hormone beta (RTH $\beta$ ). Methods Mol Biol 2018; 1801: 225-240

[8] Moran C, Agostini M, Visser WE et al. Resistance to thyroid hormone caused by a mutation in thyroid hormone receptor (TR) $\alpha 1$ and TR $\alpha 2$ : clinical, biochemical, and genetic analyses of three related patients. Lancet Diabetes Endocrinol 2014; 2: 619-626

[9] Ferrara AM, Onigata K, Ercan O et al. Homozygous thyroid hormone receptor $\beta$-gene mutations in resistance to thyroid hormone: three new cases and review of the literature. J Clin Endocrinol Metab 2012; 97: 1328-1336

[10] Dieu X, Sueur G, Moal V et al. Apparent resistance to thyroid hormones: From biological interference to genetics. Ann Endocrinol (Paris) 2019; 80: 280-285

[11] Weiss RE, Hayashi Y, Nagaya T et al. Dominant inheritance of resistance to thyroid hormone not linked to defects in the thyroid hormone receptor alpha or beta genes may be due to a defective cofactor. J Clin Endocrinol Metab 1996; 81: 4196-4203
[12] Refetoff S, DeWind LT, DeGroot LJ. Familial syndrome combining deaf-mutism, stippled epiphyses, goiter and abnormally high PBI: possible target organ refractoriness to thyroid hormone12. J Clin Endocrinol Metab 1967; 27: 279-294

[13] Uter J, Heldmann M, Rogge B et al. Patients with mutations of the thyroid hormone beta-receptor show an ADHD-like phenotype for performance monitoring: an electrophysiological study. Neurolmage Clin 2020; 26: 102250

[14] Barkoff MS, Kocherginsky M, Anselmo ] et al. Autoimmunity in patients with resistance to thyroid hormone. J Clin Endocrinol Metab 2010; 95 : 3189-3193

[15] Guerra-argüero LM, Gutiérrez-saucedo JA, Gómez-coello A et al. Resistencia a hormonas tiroideas y tiroiditis de Hashimoto. Cir Cir 2011; 453-457

[16] Choi J-H, Cho JH, Kim JH et al. Variable clinical characteristics and molecular spectrum of patients with syndromes of reduced sensitivity to thyroid hormone: genetic defects in the THRB and SLC16A2 genes. Horm Res Paediatr 2018; 90: 283-290

[17] Vela A, Pérez-Nanclares G, Ríos I et al. Thyroid hormone resistance from newborns to adults: a Spanish experience. J Endocrinol Invest 2019; 42: 941-949

[18] Xiao X, Lv C, Zhu T et al. Thyroid hormone resistance and the value of genetics: Three case reports. Medicine (Baltimore) 2019; 98: e14675

[19] Jackowski T, Petriczko E, Horodnicka-józwa A et al. Thyroid hormone resistance syndrome - own experiences. Pediatr Endocrinol Diabetes Metab 2017; 23: 209-214

[20] Rivas AM, Lado-Abeal J. Thyroid hormone resistance and its management. Vol. 29, Proceedings (Baylor University. Medical Center) 2016; 209-211

[21] Zaig E, Cohen-Ouaknine O, Tsur A et al. Clinical and molecular characteristics of eight Israeli families with thyroid hormone receptor beta mutations. Isr Med Assoc J 2018; 20: 679-686

[22] Toumba M, Neocleous V, Fanis P et al. Phenotype variability and different genotype of four patients with thyroid hormone resistance syndrome due to variants in the THRB gene. Hippokratia 2019; 23: 135-139

[23] Pappa T, Anselmo ], Mamanasiri S et al. Prenatal diagnosis of resistance to thyroid hormone and its clinical implications. J Clin Endocrinol Metab 2017; 102: 3775-3782

[24] Srichomkwun P, Anselmo ], Liao X-H et al. Fetal exposure to high maternal thyroid hormone levels causes central resistance to thyroid hormone in adult humans and mice. J Clin Endocrinol Metab 2017; 102: $3234-3240$

[25] Concolino P, Costella A, Paragliola RM. Mutational landscape of resistance to thyroid hormone beta (RTHß). Mol Diagn Ther 2019; 23: 353-368

[26] Ramos LS, Kizys MML, Kunii IS et al. Assessing the clinical and molecular diagnosis of inherited forms of impaired sensitivity to thyroid hormone from a single tertiary center. Endocrine 2018; 62: 628-638

[27] Sun H, Cao L, Zheng R et al. Update on resistance to thyroid hormone syndrome $\beta$. Ital J Pediatr 2020; 46: 168

[28] Groeneweg S, Peeters RP, Visser T] et al. Therapeutic applications of thyroid hormone analogues in resistance to thyroid hormone (RTH) syndromes. Mol Cell Endocrinol 2017; 458: 82-90

\section{Notice}

This article was changed according to the following Erratum on February $23^{\text {rd }} 2022$.

\section{Erratum}

In this article the authors names were not displayed correctly. Correct are: Maria Angeles Santos Mata, Ana Belen Ariza Jimenez, Francisco Macias Lopez and Carmen de la Camara Moraño. 